International Journal of Pure and Applied Mathematics

Volume 97 No. 3 2014, 263-271

ISSN: 1311-8080 (printed version); ISSN: 1314-3395 (on-line version)

url: http://www.ijpam.eu

doi: http://dx.doi.org/10.12732/ijpam.v97i3.1

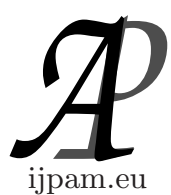

\title{
GEOMETRICAL QUATERNIONIC COUPLING FOR THREE DIMENSIONAL WAVE EQUATIONS
}

\author{
M.F. Borges ${ }^{1,2} \S$, J.A.P.F. Marão ${ }^{2}$, F.S. Costa ${ }^{3}$, J. Coelho ${ }^{3}$ \\ ${ }^{1}$ UNESP - São Paulo State University \\ S.J. Rio Preto Campus \\ 15054-000, São José do Rio Preto, BRAZIL \\ ${ }^{2}$ Department of Mathematics \\ Federal University of Maranhão - São Luís-MA \\ 65085-580, Maranhão, BRAZIL \\ ${ }^{3}$ Department of Mathematics and Informatic \\ State University of Maranhão - São Luís-MA \\ 65000-001, Maranhão, BRAZIL
}

\begin{abstract}
The present work has the scope to show the relationship between four three-dimensional waves. This fact will be made in the form of coupling, using for it the Cauchy-Riemann conditions for quaternionic functions [1], through certain Laplace's equation in [2]. The coupling will relate those functions that determine the wave as well as their respective propagation speeds.
\end{abstract}

AMS Subject Classification: 30G99, 30E99

Key Words: coupling, coupling wave equations in three dimensional space

\section{Introduction}

The study of the three-dimensional wave patterns in major physical problems

Received: December 13, 2013

(C) 2014 Academic Publications, Ltd.

$\S$ Correspondence author url: www.acadpubl.eu 
is such that the coupling of these waves can bring physical insights not yet detected. Let us consider that waves propagate with different velocities $v_{1}, v_{2}$, $v_{3}$ and $v_{4}$, and wave function given by $F_{1}, F_{2}, F_{3}$ and $F_{4}$.

To fix ideas, it will be considered a quaternionic function denoted by $F(q)$ with $q=t+x i+y j+z k$, and given by $F(q)=F_{1}+F_{2} i+F_{3} j+F_{4} k$ where the functions $F_{1}, F_{2}, F_{3}$ and $F_{4}$, are functions of the variables $t, x, y$ and $z$. The following theorem establishes the Cauchy-Riemann conditions for quaternionic functions:

Theorem 1. For any pair pontis $a$ and $b$ and any path joining them simply conect subdomain of the four-dimmensional space, the integral $\int_{a}^{b} f d q$ is independent form the given path if and only if there is a function $F=$ $F_{1}+F_{2} i+F_{3} j+F_{4} k$ such that $\int_{a}^{b} f d q=F(a)-F(b)$, and satisfying the following relations:

$$
\begin{gathered}
\frac{\partial F_{1}}{\partial t}=\frac{\partial F_{2}}{\partial x}=\frac{\partial F_{3}}{\partial y}=\frac{\partial F_{4}}{\partial z}, \\
\frac{\partial F_{2}}{\partial t}=-\frac{\partial F_{1}}{\partial x}=-\frac{\partial F_{3}}{\partial z}=\frac{\partial F_{4}}{\partial y}, \\
\frac{\partial F_{3}}{\partial t}=-\frac{\partial F_{1}}{\partial y}=-\frac{\partial F_{2}}{\partial z}=\frac{\partial F_{4}}{\partial x}, \\
\frac{\partial F_{4}}{\partial t}=\frac{\partial F_{1}}{\partial z}=-\frac{\partial F_{2}}{\partial y}=-\frac{\partial F_{3}}{\partial x} .
\end{gathered}
$$

Proof. The proof of this theorem can be analyzed in greater detail in [1].

What will be done now is the derivation of each of the above relations on each one of the variables of the problem, $t, x, y$ and $z$. Then:

$$
\begin{aligned}
& \frac{\partial^{2} F_{1}}{\partial t^{2}}=\frac{\partial^{2} F_{2}}{\partial t \partial x}=\frac{\partial^{2} F_{3}}{\partial t \partial y}=\frac{\partial^{2} F_{4}}{\partial t \partial z}, \\
& \frac{\partial^{2} F_{1}}{\partial t \partial x}=\frac{\partial^{2} F_{2}}{\partial x^{2}}=\frac{\partial^{2} F_{3}}{\partial x \partial y}=\frac{\partial^{2} F_{4}}{\partial x \partial z}, \\
& \frac{\partial^{2} F_{1}}{\partial y \partial t}=\frac{\partial^{2} F_{2}}{\partial y \partial x}=\frac{\partial^{2} F_{3}}{\partial y^{2}}=\frac{\partial^{2} F_{4}}{\partial z \partial y}, \\
& \frac{\partial^{2} F_{1}}{\partial t \partial z}=\frac{\partial^{2} F_{2}}{\partial z \partial x}=\frac{\partial^{2} F_{3}}{\partial z \partial y}=\frac{\partial^{2} F_{4}}{\partial z^{2}},
\end{aligned}
$$




$$
\begin{aligned}
& \frac{\partial^{2} F_{2}}{\partial t^{2}}=-\frac{\partial^{2} F_{1}}{\partial t \partial x}=-\frac{\partial^{2} F_{3}}{\partial t \partial z}=\frac{\partial^{2} F_{4}}{\partial t \partial y}, \\
& \frac{\partial^{2} F_{2}}{\partial t \partial x}=-\frac{\partial^{2} F_{1}}{\partial x^{2}}=-\frac{\partial^{2} F_{3}}{\partial x \partial z}=\frac{\partial^{2} F_{4}}{\partial y \partial x}, \\
& \frac{\partial^{2} F_{2}}{\partial y \partial t}=-\frac{\partial^{2} F_{1}}{\partial y \partial x}=-\frac{\partial^{2} F_{3}}{\partial y \partial z}=\frac{\partial^{2} F_{4}}{\partial y^{2}}, \\
& \frac{\partial^{2} F_{2}}{\partial z \partial t}=-\frac{\partial^{2} F_{1}}{\partial z \partial x}=-\frac{\partial^{2} F_{3}}{\partial z^{2}}=\frac{\partial^{2} F_{4}}{\partial z \partial y}, \\
& \frac{\partial^{2} F_{3}}{\partial t^{2}}=-\frac{\partial^{2} F_{1}}{\partial t \partial y}=-\frac{\partial^{2} F_{2}}{\partial t \partial z}=\frac{\partial^{2} F_{4}}{\partial t \partial x}, \\
& \frac{\partial^{2} F_{3}}{\partial t \partial x}=-\frac{\partial^{2} F_{1}}{\partial x \partial y}=-\frac{\partial^{2} F_{2}}{\partial x \partial z}=\frac{\partial^{2} F_{4}}{\partial x^{2}}, \\
& \frac{\partial^{2} F_{3}}{\partial y \partial t}=-\frac{\partial^{2} F_{1}}{\partial y^{2}}=-\frac{\partial^{2} F_{2}}{\partial z \partial y}=\frac{\partial^{2} F_{4}}{\partial y \partial x}, \\
& \frac{\partial^{2} F_{3}}{\partial t \partial z}=-\frac{\partial^{2} F_{1}}{\partial z \partial y}=-\frac{\partial^{2} F_{2}}{\partial z^{2}}=\frac{\partial^{2} F_{4}}{\partial z \partial x}, \\
& \frac{\partial^{2} F_{4}}{\partial t^{2}}=\frac{\partial^{2} F_{1}}{\partial t \partial z}=-\frac{\partial^{2} F_{2}}{\partial t \partial y}=-\frac{\partial^{2} F_{3}}{\partial t \partial x}, \\
& \frac{\partial^{2} F_{4}}{\partial t \partial x}=\frac{\partial^{2} F_{1}}{\partial x \partial z}=-\frac{\partial^{2} F_{2}}{\partial x \partial y}=-\frac{\partial^{2} F_{3}}{\partial x^{2}}, \\
& \frac{\partial^{2} F_{4}}{\partial y \partial t}=\frac{\partial^{2} F_{1}}{\partial y \partial z}=-\frac{\partial^{2} F_{2}}{\partial y^{2}}=-\frac{\partial^{2} F_{3}}{\partial y \partial x}, \\
& \frac{\partial^{2} F_{4}}{\partial t \partial z}=\frac{\partial^{2} F_{1}}{\partial z^{2}}=-\frac{\partial^{2} F_{2}}{\partial z \partial y}=-\frac{\partial^{2} F_{3}}{\partial z \partial x} .
\end{aligned}
$$

Thus, the following equations are obtained:

$$
\begin{aligned}
& \frac{\partial^{2} F_{1}}{\partial^{2} t^{2}}+\frac{\partial^{2} F_{1}}{\partial x^{2}}+\frac{\partial^{2} F_{1}}{\partial y^{2}}+\frac{\partial^{2} F_{1}}{\partial z^{2}}=0 \\
& \frac{\partial^{2} F_{2}}{\partial t^{2}}+\frac{\partial^{2} F_{2}}{\partial x^{2}}+\frac{\partial^{2} F_{2}}{\partial y^{2}}+\frac{\partial^{2} F_{2}}{\partial z^{2}}=0 \\
& \frac{\partial^{2} F_{3}}{\partial t^{2}}+\frac{\partial^{2} F_{3}}{\partial x^{2}}+\frac{\partial^{2} F_{3}}{\partial y^{2}}+\frac{\partial^{2} F_{3}}{\partial z^{2}}=0
\end{aligned}
$$

and

$$
\frac{\partial^{2} F_{4}}{\partial t^{2}}+\frac{\partial^{2} F_{4}}{\partial x^{2}}+\frac{\partial^{2} F_{4}}{\partial y^{2}}+\frac{\partial^{2} F_{4}}{\partial z^{2}}=0
$$




\section{Wave Equations}

The wave equations presents in Mathematical Physics are in the format:

$$
\frac{\partial^{2} u(t, x)}{\partial t^{2}}=c^{2} \frac{\partial^{2} u(t, x)}{\partial x^{2}}
$$

for the one-dimensional case. The three-dimensional case, is written as:

$$
\frac{\partial^{2} u(t, x, y, z)}{\partial t^{2}}=c^{2}\left(\frac{\partial^{2} u(t, x, y, z)}{\partial x^{2}}+\frac{\partial^{2} u(t, x, y, z)}{\partial y^{2}}+\frac{\partial^{2} u(t, x, y, z)}{\partial z^{2}}\right),
$$

where $c$ is the speed of wave propagation, or

$$
-\frac{1}{c^{2}} \frac{\partial^{2} u(t, x, y, z)}{\partial t^{2}}+\frac{\partial^{2} u(t, x, y, z)}{\partial x^{2}}+\frac{\partial^{2} u(t, x, y, z)}{\partial y^{2}}+\frac{\partial^{2} u(t, x, y, z)}{\partial z^{2}}=0 .
$$

\section{Coupling Equations}

Considering the equations:

$$
\begin{aligned}
& -\frac{1}{c_{1}^{2}} \frac{\partial^{2} F_{1}^{\prime}(t, x, y, z)}{\partial t^{\prime} 2}+\frac{\partial^{2} F_{1}^{\prime}(t, x, y, z)}{\partial x^{2}}+\frac{\partial^{2} F_{1}^{\prime}(t, x, y, z)}{\partial y^{2}} \\
& +\frac{\partial^{2} F_{1}^{\prime}(t, x, y, z)}{\partial z^{2}}=0 \\
& -\frac{1}{c_{2}^{2}} \frac{\partial^{2} F_{2}^{\prime}(t, x, y, z)}{\partial t^{\prime}}+\frac{\partial^{2} F_{2}^{\prime}(t, x, y, z)}{\partial x^{2}}+\frac{\partial^{2} F_{2}^{\prime}(t, x, y, z)}{\partial y^{2}}+\frac{\partial^{2} F_{2}^{\prime}(t, x, y, z)}{\partial z^{2}}=0, \\
& -\frac{1}{c_{3}^{2}} \frac{\partial^{2} F_{3}^{\prime}(t, x, y, z)}{\partial t^{\prime 2}}+\frac{\partial^{2} F_{3}^{\prime}(t, x, y, z)}{\partial x^{2}}+\frac{\partial^{2} F_{3}^{\prime}(t, x, y, z)}{\partial y^{2}}+\frac{\partial^{2} F_{3}^{\prime}(t, x, y, z)}{\partial z^{2}}=0
\end{aligned}
$$

and 


$$
\begin{aligned}
&-\frac{1}{c_{4}^{2}} \frac{\partial^{2} F_{4}^{\prime}(t, x, y, z)}{\partial t^{\prime}}+\frac{\partial^{2} F_{4}^{\prime}(t, x, y, z)}{\partial x^{2}}+\frac{\partial^{2} F_{4}^{\prime}(t, x, y, z)}{\partial y^{2}} \\
&+\frac{\partial^{2} F_{4}^{\prime}(t, x, y, z)}{\partial z^{2}}=0 .
\end{aligned}
$$

Assuming that the set of equations described in (9), (10), (11) and (12) have no physical sense, that will be reached by considering the transformation below:

$$
\frac{\partial F_{i}}{\partial t^{2}}=-\frac{1}{c_{i}^{2}} \frac{\partial F_{i}^{\prime}}{\partial t^{\prime 2}}, \quad i=1,2,3,4,
$$

where $F_{i}$ is function of variables $t^{\prime}, x, y$ and $z$. The transformation (19) makes the set of equations (5), (6), (7) and (8) to be rewritten as follows:

$$
\begin{gathered}
-\frac{1}{c_{1}^{2}} \frac{\partial^{2} F_{1}^{\prime}}{\partial t^{\prime}}=\frac{\partial^{2} F_{2}^{\prime}}{\partial t^{\prime} \partial x}=\frac{\partial^{2} F_{3}^{\prime}}{\partial t^{\prime} \partial y}=\frac{\partial^{2} F_{4}^{\prime}}{\partial t^{\prime} \partial z} \\
\frac{\partial^{2} F_{1}^{\prime}}{\partial t^{\prime} \partial x}=\frac{\partial^{2} F_{2}^{\prime}}{\partial x^{2}}=\frac{\partial^{2} F_{3}^{\prime}}{\partial x \partial y}=\frac{\partial^{2} F_{4}^{\prime}}{\partial x \partial z}, \\
\frac{\partial^{2} F_{1}^{\prime}}{\partial y \partial t^{\prime}}=\frac{\partial^{2} F_{2}^{\prime}}{\partial y \partial x}=\frac{\partial^{2} F_{3}}{\partial y^{2}}=\frac{\partial^{2} F_{4}}{\partial z \partial y}, \\
\frac{\partial^{2} F_{1}^{\prime}}{\partial t^{\prime} \partial z}=\frac{\partial^{2} F_{2}^{\prime}}{\partial z \partial x}=\frac{\partial^{2} F_{3}^{\prime}}{\partial z \partial y}=\frac{\partial^{2} F_{4}^{\prime}}{\partial z^{2}}, \\
-\frac{1}{c_{2}^{2}} \frac{\partial^{2} F_{2}^{\prime}}{\partial t^{2}}=-\frac{\partial^{2} F_{1}^{\prime}}{\partial t^{\prime} \partial x}=-\frac{\partial^{2} F_{3}^{\prime}}{\partial t^{\prime} \partial z}=\frac{\partial^{2} F_{4}^{\prime}}{\partial t^{\prime} \partial y} \\
\frac{\partial^{2} F_{2}^{\prime}}{\partial t^{\prime} \partial x}=-\frac{\partial^{2} F_{1}^{\prime}}{\partial x^{2}}=-\frac{\partial^{2} F_{3}^{\prime}}{\partial x \partial z}=\frac{\partial^{2} F_{4}^{\prime}}{\partial y \partial x} \\
\frac{\partial^{2} F_{2}^{\prime}}{\partial y \partial t^{\prime}}=-\frac{\partial^{2} F_{1}^{\prime}}{\partial y \partial x}=-\frac{\partial^{2} F_{3}^{\prime}}{\partial y \partial z}=\frac{\partial^{2} F_{4}^{\prime}}{\partial y^{2}}, \\
\frac{\partial^{2} F_{2}^{\prime}}{\partial z \partial t^{\prime}}=-\frac{\partial^{2} F_{1}^{\prime}}{\partial z \partial x}=-\frac{\partial^{2} F_{3}^{\prime}}{\partial z^{2}}=\frac{\partial^{2} F_{4}^{\prime}}{\partial z \partial y}, \\
-\frac{1}{c_{3}^{2} \frac{\partial^{2} F_{3}^{\prime}}{\partial t^{2}}}=-\frac{\partial^{2} F_{1}^{\prime}}{\partial t^{\prime} \partial y}=-\frac{\partial^{2} F_{2}^{\prime}}{\partial t^{\prime} \partial z}=\frac{\partial^{2} F_{4}^{\prime}}{\partial t^{\prime} \partial x}, \\
\frac{\partial^{2} F_{3}^{\prime}}{\partial t^{\prime} \partial x}=-\frac{\partial^{2} F_{1}^{\prime}}{\partial x \partial y}=-\frac{\partial^{2} F_{2}^{\prime}}{\partial x \partial z}=\frac{\partial^{2} F_{4}^{\prime}}{\partial x^{2}}, \\
\frac{\partial^{2} F_{3}^{\prime}}{\partial y \partial t^{\prime}}=-\frac{\partial^{2} F_{1}^{\prime}}{\partial y^{2}}=-\frac{\partial^{2} F_{2}^{\prime}}{\partial z \partial y}=\frac{\partial^{2} F_{4}^{\prime}}{\partial y \partial x} \\
\frac{\partial^{2} F_{3}^{\prime}}{\partial t^{\prime} \partial z}=-\frac{\partial^{2} F_{1}^{\prime}}{\partial z \partial y}=-\frac{\partial^{2} F_{2}}{\partial z^{2}}=\frac{\partial^{2} F_{4}^{\prime}}{\partial z \partial x},
\end{gathered}
$$




$$
\begin{gathered}
-\frac{1}{c_{4}^{2}} \frac{\partial^{2} F_{4}^{\prime}}{\partial t^{\prime}}=\frac{\partial^{2} F_{1}^{\prime}}{\partial t^{\prime} \partial z}=-\frac{\partial^{2} F_{2}^{\prime}}{\partial t^{\prime} \partial y}=-\frac{\partial^{2} F_{3}^{\prime}}{\partial t^{\prime} \partial x}, \\
\frac{\partial^{2} F_{4}^{\prime}}{\partial t^{\prime} \partial x}=\frac{\partial^{2} F_{1}^{\prime}}{\partial x \partial z}=-\frac{\partial^{2} F_{2}^{\prime}}{\partial x \partial y}=-\frac{\partial^{2} F_{3}^{\prime}}{\partial x^{2}}, \\
\frac{\partial^{2} F_{4}^{\prime}}{\partial y \partial t^{\prime}}=\frac{\partial^{2} F_{1}^{\prime}}{\partial y \partial z}=-\frac{\partial^{2} F_{2}}{\partial y^{2}}=-\frac{\partial^{2} F_{3}^{\prime}}{\partial y \partial x}, \\
\frac{\partial^{2} F_{4}^{\prime}}{\partial t^{\prime} \partial z}=\frac{\partial^{2} F_{1}^{\prime}}{\partial z^{2}}=-\frac{\partial^{2} F_{2}^{\prime}}{\partial z \partial y}=-\frac{\partial^{2} F_{3}^{\prime}}{\partial z \partial x} .
\end{gathered}
$$

\section{Concluding Remarks}

Taking only the equations that depend on time in the sets of equations (20) (23), follows that:

(i)

$$
\begin{gathered}
-\frac{1}{c_{1}^{2}} \frac{\partial^{2} F_{1}^{\prime}}{\partial t^{\prime 2}}=\frac{\partial^{2} F_{2}^{\prime}}{\partial t \partial x} ; \\
-\frac{\partial F_{4}^{\prime}}{\partial y \partial x}=-\frac{\partial^{2} F_{2}^{\prime}}{\partial t^{\prime} \partial x} ; \\
\frac{\partial^{2} F_{3}^{\prime}}{\partial t^{\prime} \partial y}=\frac{\partial^{2} F_{4}^{\prime}}{\partial t^{\prime} \partial z} \\
-\frac{\partial F_{2}^{\prime}}{\partial z \partial x}=\frac{\partial^{2} F_{4}^{\prime}}{\partial t^{\prime} \partial z}
\end{gathered}
$$

obtaining the equation:

$$
-\frac{1}{c_{1}^{2}} \frac{\partial^{2} F_{1}^{\prime}}{\partial t^{\prime 2}}-\frac{\partial^{2} F_{2}^{\prime}}{\partial t^{\prime} \partial x}-\frac{\partial^{2} F_{3}^{\prime}}{\partial t^{\prime} \partial y}+\frac{\partial^{2} F_{4}^{\prime}}{\partial t^{\prime} \partial z}=0 .
$$

Proceeding similarly, we have that:

(ii)

$$
\begin{gathered}
-\frac{1}{c_{2}^{2}} \frac{\partial^{2} F_{2}^{\prime}}{\partial t^{\prime 2}}=-\frac{\partial^{2} F_{1}^{\prime}}{\partial t^{\prime} \partial x} \\
\frac{\partial F_{4}^{\prime}}{\partial x \partial z}=\frac{\partial^{2} F_{1}^{\prime}}{\partial t^{\prime} \partial x} \\
-\frac{\partial^{2} F_{3}^{\prime}}{\partial t^{\prime} \partial z}=\frac{\partial^{2} F_{4}^{\prime}}{\partial t^{\prime} \partial y}
\end{gathered}
$$




$$
-\frac{\partial F_{3}^{\prime}}{\partial y \partial x}=\frac{\partial^{2} F_{4}^{\prime}}{\partial t^{\prime} \partial y}
$$

where, adding plots and establishing equalities, we have that:

$$
-\frac{1}{c_{2}^{2}} \frac{\partial^{2} F_{2}^{\prime}}{\partial t^{\prime 2}}+\frac{\partial^{2} F_{1}^{\prime}}{\partial t^{\prime} \partial x}-\frac{\partial^{2} F_{3}^{\prime}}{\partial t^{\prime} \partial z}-\frac{\partial^{2} F_{4}^{\prime}}{\partial t^{\prime} \partial y}=0
$$

Following the set of equations (22). We obtain that:

(iii)

$$
\begin{gathered}
-\frac{1}{c_{3}^{2}} \frac{\partial^{2} F_{3}^{\prime}}{\partial t^{\prime 2}}=-\frac{\partial^{2} F_{1}^{\prime}}{\partial t^{\prime} \partial y} \\
\frac{\partial F_{2}^{\prime}}{\partial y \partial x}=\frac{\partial^{2} F_{1}^{\prime}}{\partial t^{\prime} \partial y} \\
-\frac{\partial^{2} F_{2}^{\prime}}{\partial t^{\prime} \partial z}=\frac{\partial^{2} F_{4}^{\prime}}{\partial t^{\prime} \partial x} \\
-\frac{\partial F_{3}^{\prime}}{\partial x^{2}}=-\frac{\partial^{2} F_{4}^{\prime}}{\partial t^{\prime} \partial x}
\end{gathered}
$$

which gives us:

$$
-\frac{1}{c_{3}^{2}} \frac{\partial^{2} F_{3}^{\prime}}{\partial t^{\prime 2}}+\frac{\partial^{2} F_{1}^{\prime}}{\partial t^{\prime} \partial y}-\frac{\partial^{2} F_{2}^{\prime}}{\partial t^{\prime} \partial z}-\frac{\partial^{2} F_{4}^{\prime}}{\partial t^{\prime} \partial x}=0 .
$$

Finally, we have that:

(iv)

$$
\begin{gathered}
-\frac{1}{c_{4}^{2}} \frac{\partial^{2} F_{4}^{\prime}}{\partial t^{\prime 2}}=-\frac{\partial^{2} F_{3}^{\prime}}{\partial t^{\prime} \partial x} \\
-\frac{\partial^{2} F_{2}^{\prime}}{\partial x \partial z}=\frac{\partial^{2} F_{3}^{\prime}}{\partial t^{\prime} \partial x} \\
\frac{\partial F_{1}^{\prime}}{\partial t^{\prime} \partial z}=-\frac{\partial F_{2}^{\prime}}{\partial t^{\prime} \partial y} \\
-\frac{\partial F_{1}^{\prime}}{\partial y \partial x}=\frac{\partial F_{2}^{\prime}}{\partial t^{\prime} \partial y}
\end{gathered}
$$

again adding and making the equalities, we have:

$$
-\frac{1}{c_{4}^{2}} \frac{\partial^{2} F_{4}^{\prime}}{\partial t^{\prime 2}}+\frac{\partial^{2} F_{3}^{\prime}}{\partial t^{\prime} \partial x}+\frac{\partial^{2} F_{1}^{\prime}}{\partial t^{\prime} \partial z}+\frac{\partial F_{2}^{\prime}}{\partial t^{\prime} \partial y}=0
$$


The above equations determine when a coupling between the wave equations may be considered. This coupling is done at variable time.

Considering $c_{1}=c_{2}=c_{3}=c_{4}=c$ then the following general time depending coupling equation is obtained:

$$
\begin{aligned}
-\frac{1}{c^{2}}\left(\frac{\partial^{2} F_{1}^{\prime}}{\partial t^{2}}+\right. & \left.\frac{\partial^{2} F_{2}^{\prime}}{\partial t^{\prime 2}}+\frac{\partial^{2} F_{3}^{\prime}}{\partial t^{2}}+\frac{\partial^{2} F_{4}^{\prime}}{\partial t^{\prime 2}}\right) \\
=\frac{\partial}{\partial t^{\prime}}\left(\frac { \partial } { \partial x } \left(F_{2}^{\prime}-F_{1}^{\prime}+F_{4}^{\prime}-\right.\right. & \left.F_{3}^{\prime}\right)+\frac{\partial}{\partial y}\left(F_{3}^{\prime}-F_{1}^{\prime}+F_{4}^{\prime}-F_{2}^{\prime}\right) \\
& \left.+\frac{\partial}{\partial z}\left(-F_{4}^{\prime}+F_{3}^{\prime}+F_{2}^{\prime}-F_{1}^{\prime}\right)\right) .
\end{aligned}
$$

\section{Conclusion}

The work succeeded in establishing in a single equation the coupling between three-dimensional waves. The problem posed in this paper can be applied in the following areas of physics:

(i) Quantum Mechanics;

(ii) Electromagnetism (in the treatment of electromagnetic waves);

Therefore, we believe the formula (28) is suitable for coupling waves in space.

\section{Acknowledgments}

Three of the authors (J. Marão; F. S. Costa and M. F. Borges) have been partially supported by FAPEMA a Scientific Research Agency of the Marahnão State (BRAZIL), through research grants.

\section{References}

[1] Machado, J.M, Borges, M.F. New Ramarks on the Differenciability of Hypercomplex Functions, In: International Journal of Pure and Applied Mathematics, v. 8 n. 1 (2002), 85-101. 
[2] Marão, J. A. P. F., Borges, M. F. A Note on the Hypercomplex RiemannCauchy Like Relations for Quaternions and Laplace Equations..In: International Journal of Pure and Applied Mathematics (accept) (2013).

[3] Marão, J. A. P. F., Borges, M. F. Geometrical Hypercomplex Coupling Between Electric and Gravitational Fields.In: International Journal of Pure and Applied Mathematics (accept) (2013).

[4] Kodaira, Kunihiko, Complex Analysis, Cambridge Studies in Advanced Mathematics; Cambridge University Press Cambridge (2007) 406pp. 
